\title{
The Epicardium in Ventricular Septation During Evolution and Development
}

\author{
Robert E. Poelmann, Bjarke Jensen, Margot M. Bartelings, \\ Michael K. Richardson, and Adriana C. Gittenberger-de Groot
}

\begin{abstract}
The epicardium has several essential functions in development of cardiac architecture and differentiation of the myocardium in vertebrates. We uncovered a novel function of the epicardium in species with partial or complete ventricular septation including reptiles, birds and mammals. Most reptiles have a complex ventricle with three cava, partially separated by the horizontal and vertical septa. Crocodilians, birds and mammals, however, have completely separated left and right ventricles, a clear example of convergent evolution. We have investigated the mechanisms underlying epicardial involvement in septum formation in
\end{abstract}

\footnotetext{
R.E. Poelmann $(\varangle)$

Department of Anatomy and Embryology, Leiden University Medical Center, PO Box 9600, 2300RC Leiden, The Netherlands
}

Department of Cardiology, Leiden University Medical Center, PO Box 9600, 2300RC Leiden, The Netherlands

Institute of Biology Leiden (IBL), Leiden University, Sylvius Laboratory, Sylviusweg 72, 2333BE Leiden, The Netherlands

e-mail: R.E.Poelmann@lumc.nl

\section{B. Jensen}

Department Anatomy, Embryology and Physiology, AMC, Amsterdam, The Netherlands

Department of Bioscience-Zoophysiology, Aarhus University, Aarhus, Denmark

M.M. Bartelings

Department of Anatomy and Embryology, Leiden University Medical Center, PO Box 9600, 2300RC Leiden, The Netherlands

\section{M.K. Richardson}

Institute of Biology Leiden (IBL), Leiden University, Sylvius Laboratory, Sylviusweg 72, 2333BE Leiden, The Netherlands

A.C.G.-d. Groot

Department of Cardiology, Leiden University Medical Center, PO Box 9600, 2300RC Leiden, The Netherlands

T. Nakanishi et al. (eds.), Etiology and Morphogenesis of Congenital Heart Disease, DOI 10.1007/978-4-431-54628-3_13 
embryos. We find that the primitive ventricle of early embryos becomes septated by folding and fusion of the anterior ventricular wall, trapping epicardium in its core. This 'folding septum', as we propose to call it, develops in lizards, snakes and turtles into the horizontal septum and, in the other taxa studied, into the folding part of the interventricular septum. The vertical septum, indistinct in most reptiles, arises in crocodilians and pythonids at the posterior ventricular wall. It is homologous to the inlet septum in mammals and birds. Eventually, the various septal components merge to form the completely septated heart. In our attempt to discover homologies between the various septum components, we draw perspectives to the development of ventricular septal defects in humans.

\section{Keywords}

Evolution and development $\bullet$ Homology $\bullet$ Ventricular septum • Congenital heart disease $\cdot$ Epicardium

\subsection{Introduction}

Evolution of full division of the heart into left and right chambers by septa started with the atrium in amphibians, followed by the ventricle in amniotes. Full ventricular septation evolved independently in the lineages of the archosaurs, whose extant representatives are the birds and crocodilians and mammals [1]. Not only is evolution and development of ventricular septation of considerable biological interest, it is also of clinical relevance to the understanding of many types of ventricular septal defects in humans [2].

We demonstrated that part of the ventricular septum depends on interactions between myocardium and the epicardium including the epicardium-derived cells (EPDCs) for its development [3]. The septum will develop abnormally if the epicardium is disturbed $[4,5]$. Ventricular development, starting with a primitive common ventricular tube, leading to separation into the left and right ventricle, involves complex mechanisms including the ventricular inflow and outflow compartment $[6,7]$. Most reptile groups show a more primitive pattern comprising a horizontal and a vertical septum separating the ventricle into three interconnected cavities. How the primitive reptilian pattern was modified into the complete septum is a matter of debate. Here we report the functional role of the epicardium in ventricular septation in lizard, snake, turtle, chicken, mouse and human using descriptive and experimental approaches including quail-chicken chimeras by transplantation of quail proepicardial organ into the pericardial cavity of chicken [4]. Furthermore, epicardium-deficient animal models such as the podoplanin knockout mouse and epicardial ablation experiments in chicken embryos were investigated [4, 5]. With DiI labelling [8] of the myocardial surface in chicken embryos, the outgrowth of the cardiac compartments was analysed. Tbx5 expression showed gradients along the cardiac tube and so may be a useful marker for regionalisation of the heart [9]. For experimental details and full material and method description, we refer to [10]. 


\subsection{Septum Components in the Completely Septated Heart}

Varying terminology is used for components of the ventricular septum and their respective boundaries; we adopted the following. The posterior component of the interventricular septum, between the left and right atrioventricular junctions, is the inlet septum. We propose to use the term 'folding septum' (newly defined here) for the anterior component. The septal band is a muscular ridge on the right ventricular septal surface between the inlet and folding septum, extending as the moderator band towards the right ventricular free wall. In the completely septated heart, the aortopulmonary septum is the last component that closes the interventricular communication, but has not been specifically studied here.

\subsection{The Presence of the Epicardium in Amniotes}

Among other species we examined embryos of Macklot's python (Liasis mackloti), European pond turtle (Emys orbicularis) and Chinese soft-shell turtle (Pelodiscus sinensis). The presence of epicardium on the outer face of the myocardial heart tube is confirmed. A pronounced subepicardium in the inner curvature of the looping heart tube at the site of the bulboventricular fold is confirmed, here referred to as epicardial cushion, being almost as elaborate as the adjacent endocardial atrioventricular and outflow tract cushions (Fig. 13.1a). In chicken (Fig. 13.2a, b) and mouse embryos, the presence of the (sub)epicardium is less extensive compared to the turtle. In human embryos at Carnegie stages, 11-15 $(3.6-7 \mathrm{~mm})$, the surface of the heart is closely covered by the epicardial epithelium, whereas the inner curvature harbours an extensive epicardial cushion (Fig. 13.1b) comparable to the turtle.
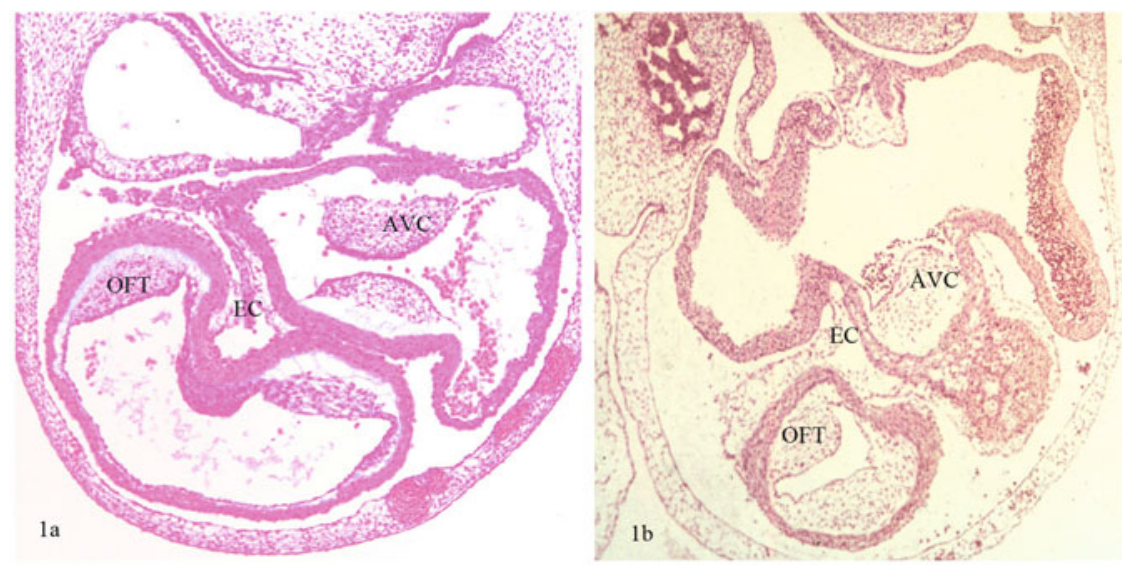

Fig 13.1 (a, b) Sections of turtle (Emys orbicularis) (a) and human embryo (b) of comparable developmental stages showing the epicardial cushion (EC) in the inner curvature of the looping heart tube. $A V C$ atrioventricular cushion, $O F T$ outflow tract cushion 

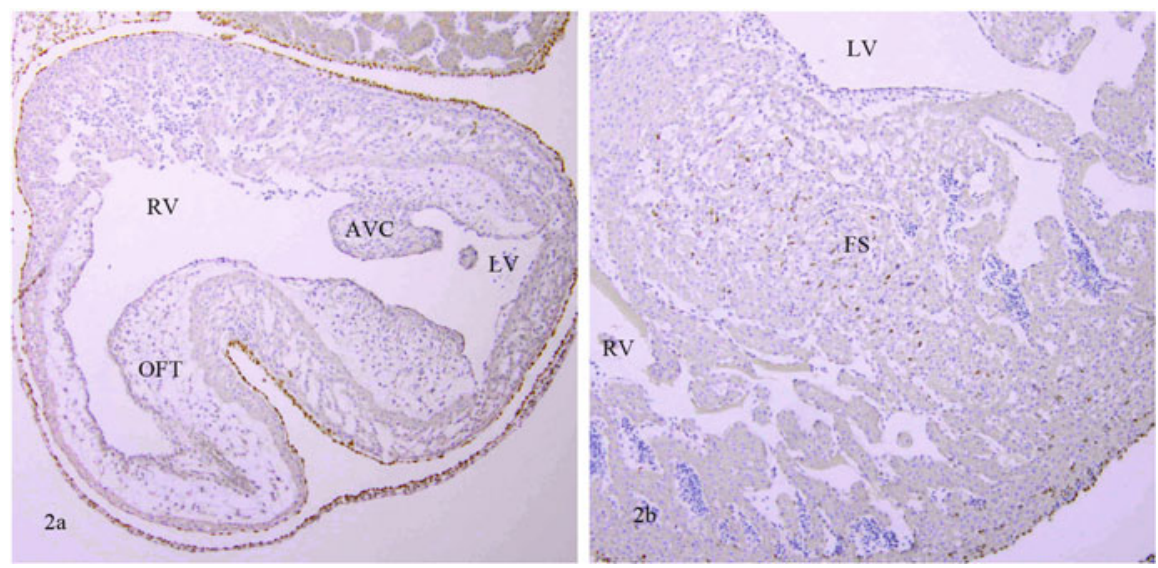

Fig 13.2 (a) Chicken embryo stage 27 showing the start of the folding septum between OFT and AVC, stained for the Wilm's tumour antigen (WT1) marking the epicardium (brown). (b) Chicken embryo stage 31 with an elaborate folding septum (FS) showing dispersed EPDCs (brown) between the cardiomyocytes. $L V$ left ventricle, $R V$ right ventricle

\subsection{The Epicardium in the Avian Heart}

To investigate the fate of the epicardium during ballooning of the ventricles and subsequent folding of the septum, we constructed quail-chicken chimeras. An isochronic quail proepicardial organ (PEO) including a small piece of adjacent liver tissue to provide endothelial cells was transplanted into the pericardial cavity of HH15-17 chick embryos in an anterior position. Antibody staining revealed that quail EPDCs and endothelial cells were present in the anterior folding septum, but not in the dorsal inlet septum (Fig. 13.3a, b). The quail epicardial sheet dispersed into individual cells that became distributed between the cardiomyocytes in later stages, mostly in the core of the folding septum. In a second set of chimeras, the quail PEO was positioned more dorsally, providing for quail EPDCs and endothelial cells on the posterior ventricular surface, subsequently migrating into the inlet septum including the septal band, without crossing over to the anterior folding septum. This indicates the inlet septum as a separate component. Furthermore, an epicardial sheet, characteristic for the folding septum, was not encountered. As a consequence, expansion of the ventricles results in anterior folding of the ventricular wall, but not of the posterior wall, probably because of the physical constraints imposed by attachment of the heart to the dorsal body wall [10].

To study septum folding during ballooning of the ventricle, fluorescent DiI was tattooed (HH15-17) onto the ventral myocardial surface and embryos were sacrificed between HH22 and 33. DiI was applied on the surface of the future fold and the dye fragmented during further development into a left-sided part, incorporated into the left side of the folding septum and a right-sided part. This 

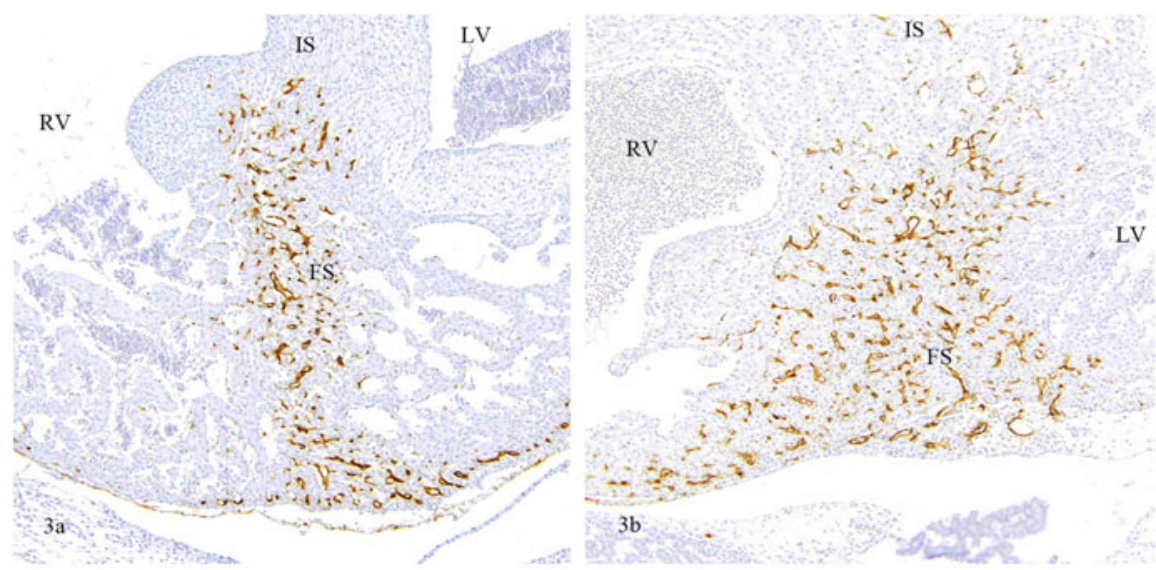

Fig 13.3 (a, b) Quail-chicken chimeras from the anterior position. The folding septum (FS) harbours quail-derived endothelial cells stained with the QH1 antibody (brown), whereas the inlet septum (IS) is virtually negative
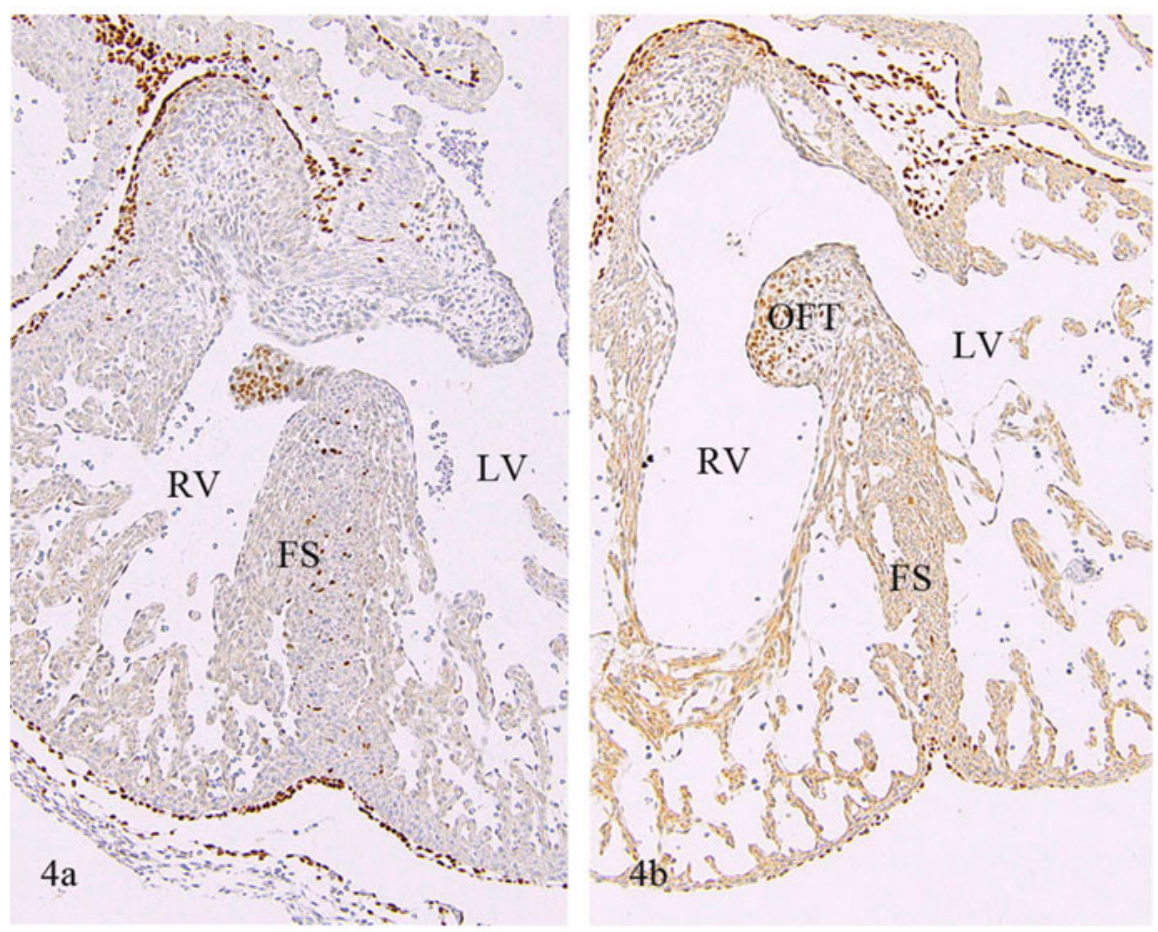

Fig 13.4 Podoplanin wild type (a) and -/- mouse (b), embryonic day 12.5. Note the diminished number of epicardial cells and EPDCs (brown in this WT1 staining) and the thin folding septum in the mutant 
Fig 13.5 DiI-labelled chicken embryo stage 31 with a thin strip of the dye in the folding septum adjacent to the right ventricular surface

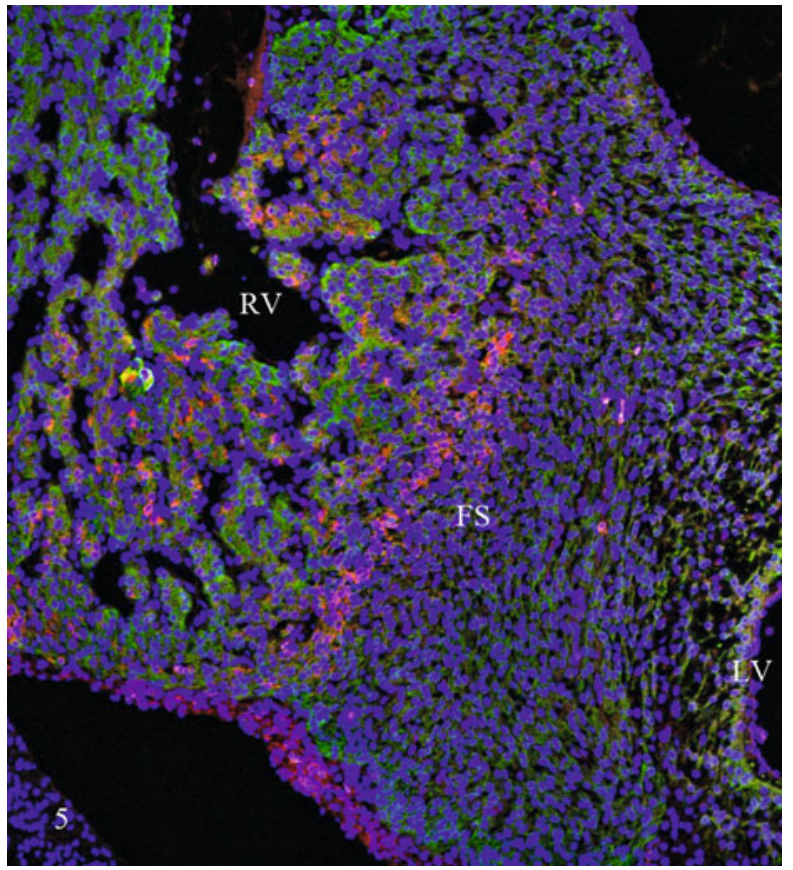

indicates a longitudinally directed morphogenetic expansion of the right ventricular wall compared to a transverse expansion of the left ventricle. At stage 31 the dye was completely embedded in the folding septum as a narrow fluorescent strip (Fig. 13.5) markedly close to the right ventricular face of the septum, indicative of a less elaborate right ventricular contribution to the septum [10].

\subsection{Disturbance of the Epicardium}

Deficient septation is known in several mouse mutants and we have analysed the podoplanin mutant mouse. Podoplanin has multiple roles in the maintenance of the epithelium and is expressed in the mesothelium of the body cavities including the epicardium. The knockout mouse presents with an underdeveloped PEO and abnormal epicardial covering resulting in only a few EPDCs [5]. The phenotype shows multiple malformations including an atrioventricular septum defect. At embryonic day 12.5, the myocardium and the folding septum are very thin, and the inlet septum is spongy. The diminutive septum is nearly devoid of EPDCs suggestive of an instructive role for these cells in septation (Fig. 13.4a, b). 


\subsection{Septum Components in Reptilian Hearts}

The horizontal septum of developing reptiles is found in comparable stages and location, separating ventricular cavities and harbouring an epicardial sheet similar to the folding septum in mammals and birds. We prefer to address this structure as 'folding septum'. The presence and extent of the vertical septum (homologous to inlet septum) differs among reptiles, being virtually absent in turtles and most non-crocodilian reptiles, but more prominent in varanids and pythonidae [11]. In the python myocardial apical trabeculations traverse the ventricle to partly separate the cavum pulmonale from the cavum arteriosum.

\subsection{Tbx5 Expression Patterns}

The transcription factor Tbx 5 is implicated in full ventricular septation in mammals and is expressed strongly in the left part of the cardiac tube, with expression declining towards the right side [9]; we confirmed this expression pattern also in early stages of the copperhead rat snake, Coelognathus. Slightly later in the development, we show that embryonic turtle and python show expression of Tbx5 that declines at the folding septum, but not over the dorsal wall, where a sharp decline was found only near the outflow tract. In the chicken the $T b \times 5$ mRNA gradient identifies the right ventricle (weak expression) from the outflow tract (negative expression). Furthermore, a Tbx5 decline is found at the folding septum showing only strong left-sided expression, whereas $T b x 5$ expression is present on both sides of the inlet septum including the septal band. Thus, in the chicken the folding and inlet septa are differentially identified by Tbx 5 gradients. In the mouse two Tbx 5 gradients identify the inlet and folding septum. Protein expression is strongest in the trabeculations of the left ventricle, weaker in the right ventricular inlet including septal band and weakest to negative in the right ventricular outflow.

\subsection{Discussion}

All amniotes exhibit a folding (horizontal) septum, and here we showed the involvement of the epicardium in fusion of the two opposing myocardial walls. In mammals and birds, the folding septum forms the anterior part of the definitive septum. Reducing the size of the PEO leads to diminished or retarded covering of the myocardium and diminished production of EPDCs $[4,5,12]$. The latter can differentiate into smooth muscle cells of the coronary vessels and into perivascular and interstitial fibroblasts [13]. It is evident that mechanical or genetic interference with the epicardium or EPDCs not only disturbs coronary vascularisation but can also strongly influence cardiomyocyte differentiation and ventricular septation.

Different septal components [6, 7] have been identified in the completely septated hearts which have been the subject of continued debate also involving exploration of the position of central muscular ventricular septal defects and the 
connection of the tricuspid valve tendinous cords related to either septal components. It was generally agreed that the septal band belonged to the primary septum being the anterior or, as we now refer to it, the folding component of the septum, but we demonstrated that it belongs to the posterior inlet septum. We newly identified the border between inlet and folding component using several criteria: (1) the atrioventricular cushions connect to the inlet component including the septal band; (2) the tip of the septal outflow tract cushion originates where the folding septum merges with the inlet septum; (3) quail cells derived from posterior PEO chimeras do not crossover from inlet septum including septal band to the folding septum, and vice versa in anterior chimeras, there is no crossover from folding to inlet septum; and (4) Tbx5 expression, in contrast to earlier publications [9, 14], is strong on the left and right side of the posteriorly located inlet septum including the septal band, whereas only left-sided positivity is found at the anterior folding septum. From this we postulate that the avian and mammalian embryonic ventricle (homologous to the cavum dorsale of reptiles, which is the cavum arteriosum and venosum combined) give rise to both the left ventricle and the inlet of the right ventricle. This is an indication that the inlet septum originates in its entirety from the wall of the primitive ventricle, the cavum dorsale. In the right ventricle, the boundary of the inlet septum is provided by the septal band, which is not present in the left ventricle, leaving here the boundary between the inlet and folding septum less well determined. Our new model for separating the ventricles implicates more than one component and has consequences for understanding the development of central muscular ventricular septal defects. (Note: The development of the membranous and the outflow tract septum has not been specifically addressed in this study) Interestingly, elephants and some relatives including seacows show a very deep anterior interventricular sulcus [10]. The anatomy of the right ventricular septal band and the attachment of the tricuspid chordae tendineae suggest a diminished folding mechanism resulting in retention of an early embryonic state, also known as neoteny.

In conclusion, we have explored an evo-devo context for the hitherto overlooked role for the epicardium in septation and have clarified complex homologies in amniotes of the ventricular septum to understand clinical disorders of the heart in humans.

Acknowledgements B.J. is supported by the Danish Council for Independent Research I Natural Sciences and M.K.R. is supported by AgentschapNL, Smartmix SSM06010.

Open Access This chapter is distributed under the terms of the Creative Commons AttributionNoncommercial 2.5 License (http://creativecommons.org/licenses/by-nc/2.5/) which permits any noncommercial use, distribution, and reproduction in any medium, provided the original author(s) and source are credited.

The images or other third party material in this chapter are included in the work's Creative Commons license, unless indicated otherwise in the credit line; if such material is not included in the work's Creative Commons license and the respective action is not permitted by statutory regulation, users will need to obtain permission from the license holder to duplicate, adapt or reproduce the material. 


\section{References}

1. Holmes EB. A reconsideration of the phylogeny of the tetrapod heart. J Morphol. 1975;147:209-28.

2. Jacobs JP, et al. Congenital heart surgery nomenclature and database project: atrial septal defect. Ann Thorac Surg. 2000;69:S18-24.

3. Gittenberger-de Groot AC, et al. Epicardium-derived cells contribute a novel population to the myocardial wall and the atrioventricular cushions. Circ Res. 1998;82:1043-52.

4. Lie-Venema $\mathrm{H}$, et al. Origin, fate, and function of epicardium-derived cells (EPCDs) in normal and abnormal cardiac development. Sci World J. 2007;7:1777-98.

5. Mahtab EAF, et al. Cardiac malformations and myocardial abnormalities in podoplanin knockout mouse embryos: correlation with abnormal epicardial development. Dev Dyn. 2008;237:847-57. doi:10.1002/dvdy.21463.

6. Wenink ACG. Embryology of the ventricular septum. Separate origin of its components. Virchows Arch. 1981;390:71-9.

7. van Mierop LH, Kutsche LM. Development of the ventricular septum of the heart. Heart Vessels. 1985;1:114-9.

8. Darnell DK, et al. Dynamic labeling techniques for fate mapping, testing cell commitment, and following living cells in avian embryos. Methods Mol Biol. 2000;135:305-21.

9. Koshiba-Takeuchi K, et al. Reptilian heart development and the molecular basis of cardiac chamber evolution. Nature. 2009;461:95-8. doi:10.1038/nature08324.

10. Poelmann RE et al. Evolution and development of ventricular septation in the amniote heart. PLoS ONE 2014;9(9):e106569. doi:10.1371/journal.pone.0106569

11. Jensen B, et al. Structure and function of the hearts of lizards and snakes. Biol Rev Camb Philos Soc. 2014;89:302-36.

12. Gittenberger-de Groot AC, et al. Epicardial outgrowth inhibition leads to compensatory mesothelial outflow tract collar and abnormal cardiac septation and coronary formation. Circ Res. 2000;87:969-71.

13. Gittenberger-de-Groot AC, et al. The arterial and cardiac epicardium in development, disease and repair. Differentiation. 2012;84:41-53. doi:10.1016/j.diff.2012.05.002.

14. Greulich F, Rudat C, Kispert A. Mechanisms of T-box function in the developing heart. Cardiovasc Res. 2011;91:212-22. doi:10.1093/cvr/cvr112. 